Supporting Information

\title{
A Single Molecule Polyphenylene-Vinylene Photonic Wire
}

Mikael Madsen, Mette R. Bakke, Daniel A. Gudnason, Alexander F. Sandahl, Rikke A. Hansen, Jakob B. Knudsen, Anne Louise B. Kodal, Victoria Birkedal,* Kurt V. Gothelf* 


\section{S1. General Information}

Poly(APPV-DNA) was prepared according to the previously published protocol. ${ }^{1}$ Fluorophore-labeled staple strands (with Atto427 and Atto594) were prepared from amino-modified oligonucleotides obtained from IDT and fluorophore NHS-esters purchased from Sigma Aldrich. The fluorophore-labeled strands were purified by reversed phase HPLC on an Agilent 1100 or 1200 system equipped with a 3u Phenomenex Clarity RP-oligo (50 $\mathrm{mm} \times 4.6 \mathrm{~mm}$ ) column running a gradient of acetonitrile in $0.1 \mathrm{M}$ triethylammonium acetate (TEAA). The products were characterized using LC-MS on a Shimadzu LCMS-2020EV connected to a Shimadzu Prominence RP-UPLC system equipped with a Kinetex 2.6u EVO C18 column $(50 \times 3.0 \mathrm{~mm})$ from Phenomenex running a gradient of $\mathrm{MeOH}$ in 1,1,1,3,3,3-hexafluoroisopropanol(HFIP)/trimethylamine(TEA) (HFIP, $100 \mathrm{mM} / \mathrm{TEA}, 8 \mathrm{mM}$ ). For all experiments, milliQ water was obtained from a Milli-Q Biocel system from Millipore or distilled water from a Glas bi-distiller from GFL. The M13mp18 scaffold strand was purchased from New England Biotech, and unmodified, amino-modified and biotinylated staple strands were purchased from IDT or Sigma Aldrich. All fluorescence spectra were recorded on a Fluoromax-4 fluorimeter from Horiba using $60 \mu \mathrm{L}$ Hellma Quartz Suprasil cuvettes $(3 \times 3 \mathrm{~mm})$ and analyzed using AE freeware. ${ }^{2}$ AFM imaging was performed in tapping mode on freshly cleaved mica in liquid on an Agilent 5500 AFM using Picoview software, and the images were analyzed using Gwyddion software. Single molecule Fluorescence was recorded using an ONI nanoimager S microscope and data analysed with the iSMS software. ${ }^{3}$

\section{S2. DNA origami design}

The twist-corrected rectangular DNA origami design utilized in these studies is based on the design used in our previous studies, ${ }^{3}$ and except for the modified staple strands, the sequences are identical. Table S1 shows the sequences used for fluorophore modification and figure S1 shows a schematic overview of the incorporation of the fluorophore-labeled strands. Table S2 shows the staple strands required for immobilization of the origami structure on quartz slides through biotinylated corners. These strands replace some of the staple strands in the original design.

\begin{tabular}{|l|l|l|l|l|}
\hline Name & $5^{\prime}$ & $3^{\prime}$ & Sequence $\left(5^{\prime}-3^{\prime}\right)$ & $\begin{array}{l}\text { Included } \\
\text { in setup }\end{array}$ \\
\hline A & $16[159]$ & $18[160]$ & /5AmMC6/CTTTACCTAATTTGCCTGTTAATCGCAAGACAAAGCATAGGTC & All \\
\hline D1 & $13[176]$ & $11[175]$ & /5AmMC6/CTTTACCTAATTTTTTCCTTAGCAAGCAAATCAGATATATTTTGC & 1 \\
\hline D1' & $19[176]$ & $17[175]$ & /5AmMC6/CTTTACCTAATTTGTGAATAAGTGAATTTATCAAAATAACGCGAG & 1 \\
\hline D2 & $12[159]$ & $14[160]$ & CTTTACC/iAmMC6T/AATTTTTATCCGAAACCAATCAATAATCAGAACGC & $1-2$ \\
\hline D2' & $20[159]$ & $22[160]$ & CTTTACC/iAmMC6T/AATTTAAACAAATCAGGTTTAACGTCACTGAATAA & $1-2$ \\
\hline D3 & $11[176]$ & $9[175]$ & CTTTACC/iAmMC6T/AATTTACCCAGCTCAAATAAGAAACGATTCCTGAACA & $1-3$ \\
\hline D3' & $21[176]$ & $19[175]$ & CTTTACC/iAmMC6T/AATTTTACAGTAATTACCTGAGCAAAAGAATATGTGA & $1-3$ \\
\hline D4 & $10[159]$ & $12[160]$ & CTTTACC/iAmMC6T/AATTTAACGTCAATCAAGATTAGTTGCTAGAAGGC & $1-4$ \\
\hline
\end{tabular}




\begin{tabular}{|l|l|l|l|l|}
\hline D4' & $22[159]$ & $24[160]$ & CTTTACC/iAmMC6T/AATTTTGGAAGGACGTTATTAATTTTAAATAGATA & $1-4$ \\
\hline D5 & $9[176]$ & $7[175]$ & CTTTACC/iAmMC6T/AATTTAAGTCAGAACCGAAGCCCTTTTTACTCCTTAT & $2-5$ \\
\hline D5' & $23[176]$ & $21[175]$ & CTTTACC/iAmMC6T/AATTTAGTAACATTGTTTGGATTATACTTGATGAATA & $2-5$ \\
\hline D6 & $8[159]$ & $10[160]$ & CTTTACC/iAmMC6T/AATTTAAGCAGAGAATTAACTGAACACTTTTGTTT & $3-6$ \\
\hline D6' & $24[159]$ & $26[160]$ & CTTTACC/iAmMC6T/AATTTATACATTTCTAAAGCATCACCTAGATAAAA & $3-6$ \\
\hline D7 & $7[176]$ & $5[175]$ & CTTTACC/iAmMC6T/AATTTTACGCAGTGAAAATTCATATGGTTGTCACCGA & $4-6$ \\
\hline D7' & $25[176]$ & $23[175]$ & CTTTACC/iAmMC6T/AATTTCTCAAATAATAGATTAGAGCCGTCAAAGTTTG & $4-6$ \\
\hline D8 & $6[159]$ & $8[160]$ & CTTTACC/iAmMC6T/AATTTCCAAAGACTGGCATGATTAAGAAGAAAAGT & $5-6$ \\
\hline D8' & $26[159]$ & $28[160]$ & CTTTACC/iAmMC6T/AATTTCAGAGGTCCAACAGAGATAGAAACGCTCAA & $5-6$ \\
\hline D9 & $5[176]$ & $3[175]$ & CTTTACC/iAmMC6T/AATTTCTTGAGCCTCAGTAGCGACAGAATATCACCGG & 6 \\
\hline D9' & $27[176]$ & $25[175]$ & CTTTACC/iAmMC6T/AATTTACCTGAAAACGAACCACCAGCAGATGCTGAAC & 6 \\
\hline
\end{tabular}

Table S1. Sequences of amino-modified staple strands used for incorporation of fluorophore labels. The outer right column designates which setups include the respective strands. The oligo names correspond to the positions marked in figure S1. The part of the sequences which are complementary to the DNA on poly(APPV-DNA) is shown in green, 3T spacers are shown in blue and the staple sequences are shown in black. /5AmMC6/ and /iAmMC6T/ are commercial codes from Integrated DNA Technologies, Inc. (IDT) and corresponds to a six carbon 5' amino modifier and a six carbon spacer with a terminal amine attached to a thymine base through an amide bond, respectively. For the acceptor (A) strand either an Atto594 or Alexa647 (in the case of single molecule studies) dye was attached to the amine, while an Atto425 dye was used for the donor (D) strands. Fluorophores were coupled onto the amine functionalized DNA by NHS-chemistry as described in section S3.

\begin{tabular}{|l|l|l|l|}
\hline Name & $\mathbf{5}^{\prime}$ & $\mathbf{3}^{\prime}$ & Sequence $\mathbf{( 5}^{\prime}$-3') \\
\hline Ft.SS.BottomRightBiotin & $29[192]$ & $31[191]$ & /5Biosg/TTGAGTAGACCGATTAAAGGGATTTGGGCGCGT \\
\hline Ft.SS.TopRightBiotin & $3[192]$ & $5[191]$ & /5Biosg/TGGAACCGCGATAGCAGCACCGTAAATTTGGGA \\
\hline Ft.SS.TopLeftBiotin & $2[47]$ & $0[48]$ & /5Biosg/TTCTAAAGTTCAGAACCGCCACCCTTTGATATA \\
\hline Ft.SS.BottomLeftBiotin & $28[47]$ & $26[48]$ & /5Biosg/TTGCGTTGCTCCCCGGGTACCGAGCCCAGGGT \\
\hline Ft.SS.TopLeft1NB & $0[47]$ & $2[32]$ & AGTATAGCCCGGAATAGGTGTATTCAGAACCGCCACCCTTTGTCGT \\
\hline Ft.SS.TopLeft2NB & $5[48]$ & $2[48]$ & GCTTGCAGATTTCTTAAACAGCTTCAACAGTTTCAGCGGACGTAACGA \\
\hline Ft.SS.TopRight1NB & $0[191]$ & $3[191]$ & TTAATGCCTCATACATGGCTTTTGAGAACCACCACCAGAGCCACCACC \\
\hline Ft.SS.BottomLeft2NB & $31[48]$ & $28[48]$ & CCAGTTTGCGAAAATCCTGTTTGACGCGCGGGGAGAGGCGACATTAAT \\
\hline Ft.SS.BottomLeft1NB & $26[47]$ & $23[47]$ & TTCCCAGTTTCTGGTGCCGGAAACGTGCATCTGCCAGTTTTTCGCGTC \\
\hline Ft.SS.TopRight2NB & $5[192]$ & $8[192]$ & ATTAGAGCTTTGTCACAATCAATAATGTTAGCAAACGTAGTAGCAATA \\
\hline Ft.SS.BottomRight1NB & $26[191]$ & $29[191]$ & AATACCGAGCGTAAGAATACGTGGGGAAAAACGCTCATGGCACTTGCC \\
\hline Ft.SS.BottomRight2NB & $31[192]$ & $29[207]$ & ACTATGGTTGCTTTGACGAGCACGGGAGCTAAACAGGAGGAGAACTCA \\
\hline
\end{tabular}


Table S2. Staple strands required for origami structure with biotinylated corners. These are required for immobilization of the structures onto quartz slides in order to perform single molecule studies. 


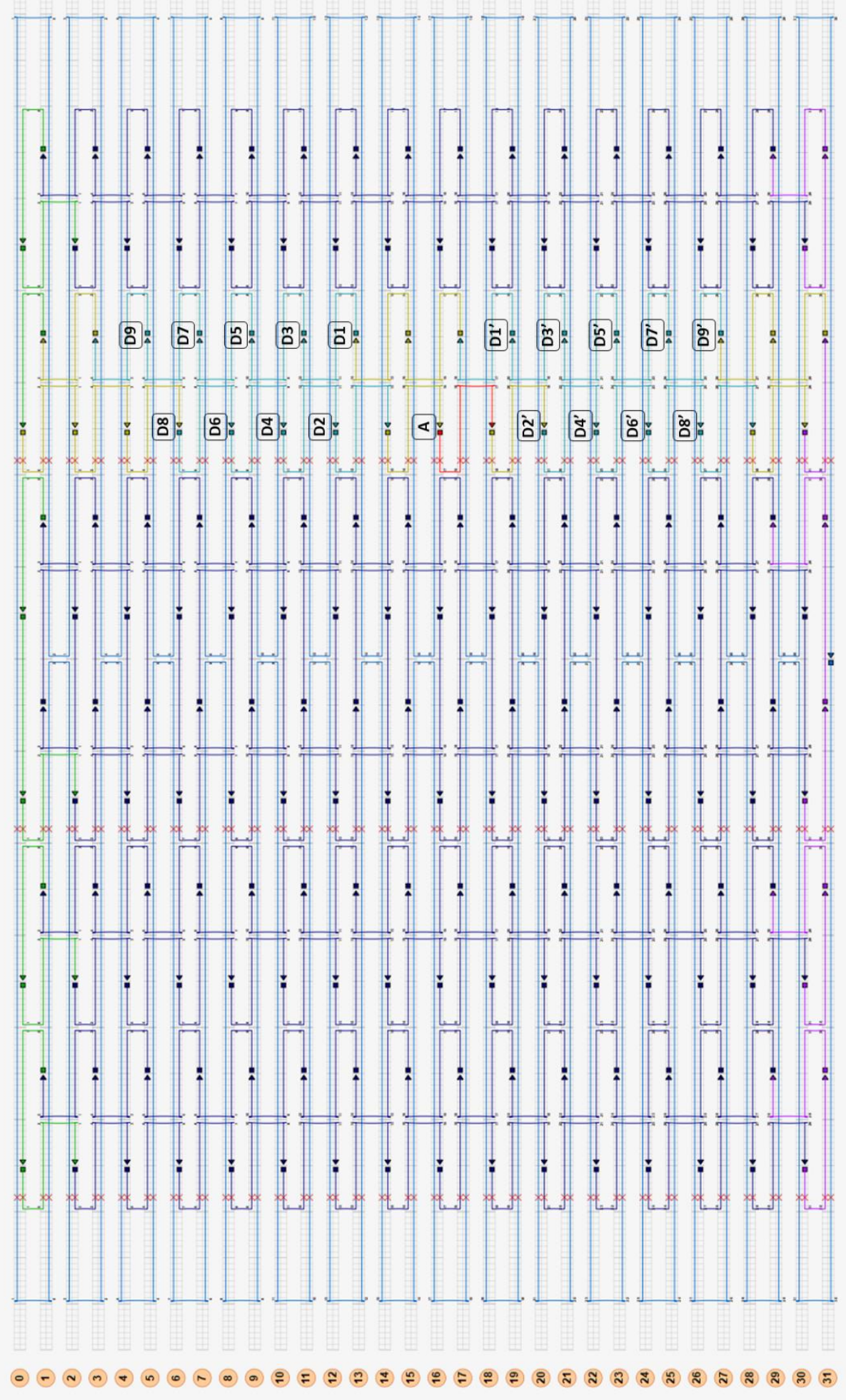

Figure S1. Overview of the origami design from the CaDNAno file with fluorophore positions marked corresponding to the nomenclature given in Table S1. A designates the acceptor, whereas D and D' designates donor pairs. 


\section{S3. Fluorescence spectroscopy measurements}

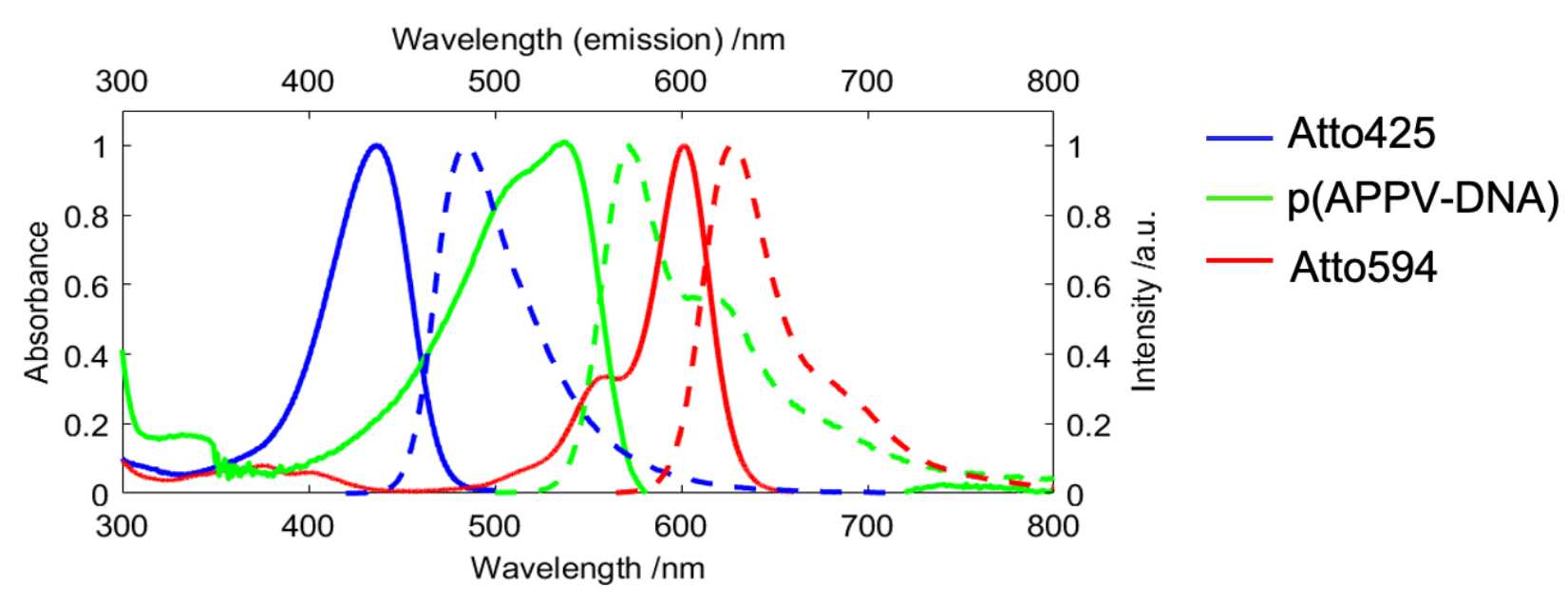

Figure S2. The absorption and emission spectra of Atto425, APPV-DNA and Atto594

For ensemble fluorescence measurements, fluorescence spectra of all samples were recorded upon excitation at three different wavelengths:

$D_{\text {exc }}=425 \mathrm{~nm}$, Emission: $435-750 \mathrm{~nm}$, that mostly excites the donor fluorophores (Figure S2).

$P_{\text {exc }}=500 \mathrm{~nm}$, Emission: $510-750 \mathrm{~nm}$, that mostly excites poly(APPV-DNA).

$A_{\text {exc }}=575 \mathrm{~nm}$, Emission: 585-750 nm for direct excitation of the Atto594 acceptor

For samples having Alexa647 as acceptor, we used $A_{\text {exc }}$ (Alexa647) $=600 \mathrm{~nm}$.

The spectra were recorded at room temperature using an excitation slit size of $4 \mathrm{~nm}$, and an emission slit size of $3 \mathrm{~nm}$. Background spectra for buffer alone were subtracted in all experiments. Measurements were made in triplicates. 


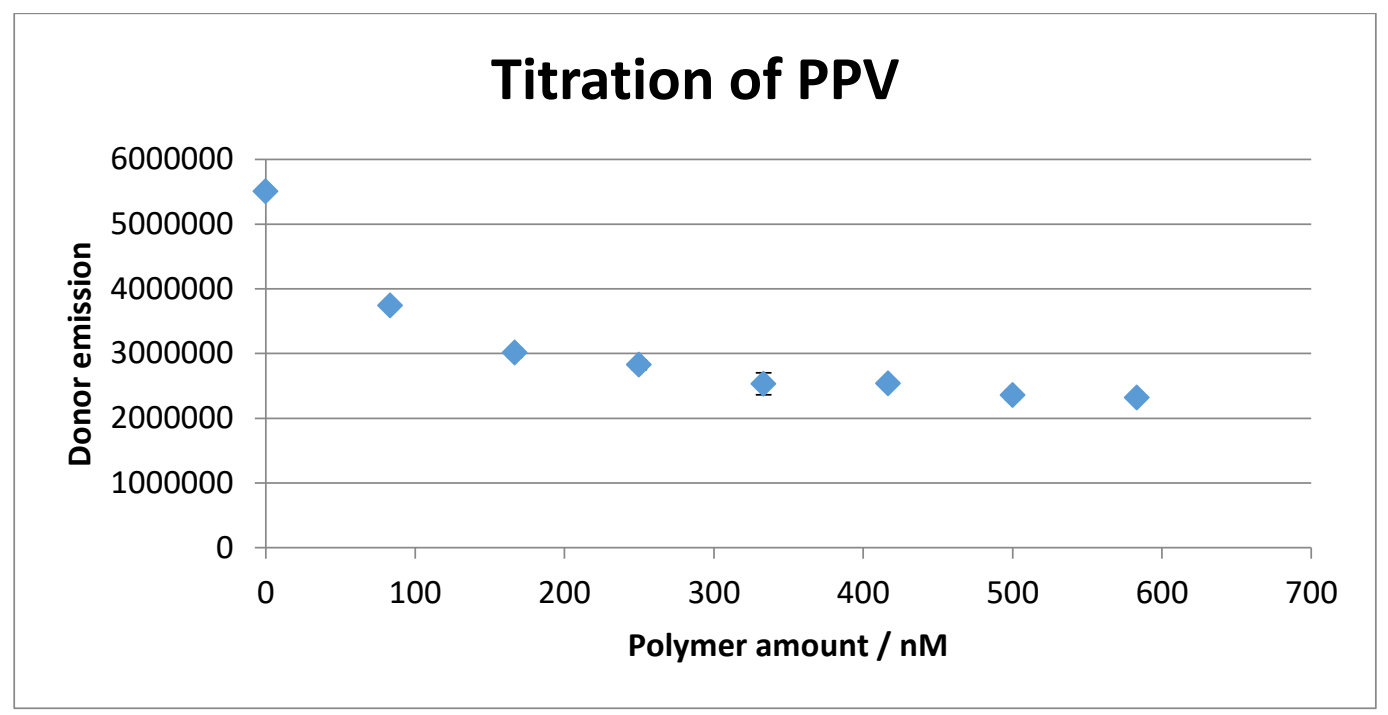

Figure S3: Screening of ratio between the origami platform containing eight donor fluorophores and poly(APPVDNA). The concentration of origami was kept constant at $2.5 \mathrm{nM}$, while the amount of polymer is given as the concentration of DNA added. The integral of the donor component was found for each sample and plotted as a function of the amount of polymer added. A mean of duplicate measurements is shown with the standard deviation (only one measurement for sample without polymer).

\section{S4. Fluorescence spectroscopy data analysis}

All spectra containing more than one component were decomposed into the component spectra using the spectral decomposition tool of the AE software. To achieve this a spectrum of each component is needed. The donor components (Figure S4, top) were obtained from samples only containing donors on origami upon $D_{\text {exc. }}$. The polymer components (Figure S4, middle) were obtained from samples only containing poly(APPV-DNA) on origami upon $\mathrm{D}_{\mathrm{exc}}$ or $\mathrm{P}_{\mathrm{exc}}$. The acceptor components (Figure $\mathrm{S4}$, bottom) were obtained from samples containing poly(APPV-DNA) and acceptor on origami upon $D_{\text {exc }}$ or $P_{\text {exc }}$ by normalizing the spectra at the maximum polymer emission wavelength and subtracting the polymer contribution to the spectrum. This method was used for obtaining the acceptor component because a slight shift in the emission was observed for the acceptor in the presence of poly(APPV-DNA). The spectral decomposition enabled reporting fluorescence intensities of individual components by integration over its spectrum, which allowed quantification of the data and investigation of energy transfer efficiencies.

The spectrally decomposed acceptor emission following donor excitation (425 nm) $(I(A)$, ) contains contributions from direct excitation of the acceptor $I(A)_{\text {direct }}$, donor excited acceptor emission $\left(I(A)_{D \rightarrow A}\right)$, polymer excited acceptor emission $\left(I(A)_{P \rightarrow A}\right)$ and acceptor emission from energy transferred from the donor through the polymer $\left(I(A)_{D \rightarrow P \rightarrow A}\right)$. To quantify energy transfer, we first determined $\left(I(A)_{D \rightarrow P \rightarrow A}\right)$ using $I(A)$, 
$I(A)_{\text {direct }}, I(A)_{D \rightarrow A}$ and $I(A)_{P \rightarrow A}$ obtained from different control samples from spectra obtained following donor excitation at $425 \mathrm{~nm}$.

The parameter $I(A)_{\text {direct }}$, was found using DNA origami sample containing only an acceptor. $I(A)_{D \rightarrow A}$ was found using a DNA origami sample containing donors and an acceptor and no polymer. This quantity was found to be negligible (Figure S5). Lastly, $I(A)_{P \rightarrow A}$ was determined using DNA origami samples containing polymer and acceptor. In the latter conditions the polymer emission bleeds into the same spectral area as the acceptor emission. The acceptor signal was obtained by deconvoluting emission spectra of samples containing poly(APPVDNA) and acceptor when excited at $575 \mathrm{~nm}$. We further determined the total donor emission, $I(D)_{\text {notransfer }}$, using DNA origami samples containing only donors and used data from DNA origami samples containing only polymers, donors and polymers, polymers and acceptors or only acceptors to determine the energy transfer efficiency from the donors to the polymer, $E_{D \rightarrow P}$ and from the polymer to the acceptor $E_{P \rightarrow A}$ :

$E_{D \rightarrow P}(\%)=\frac{I(P)_{D P}-I(P)_{P}}{I(D)_{\text {no transfer }}} \frac{\Phi_{D}}{\Phi_{P}} 100 \%$

and

$E_{P \rightarrow A}(\%)=\frac{I(A)_{P A}-I(A)_{A}}{I(P)_{\text {no transfer }}} \frac{\Phi_{P}}{\Phi_{A}} 100 \%$

where $I(P)_{D P}$ and $I(P)_{P}$ are fluorescence intensitities arising from the polymer in DNA origami samples where donors and polymers are present or with only polymers, respectively and $I(A)_{P A}$ and $I(A)_{A}$ are acceptor fluorescence intensitities from DNA origami samples containing polymers and acceptors or acceptors only. $\Phi_{X}$ is the quantum yield of the different emitting species (polymer: $\mathrm{X}=\mathrm{P}$, acceptor: $\mathrm{X}=\mathrm{A}$ or donor: $\mathrm{X}=\mathrm{D}$ ), with $\Phi_{D}=$ $0.9, \Phi_{A}=0.85$ (reference: ATTO-TEC Catalogue 2009/2010 p.24) and $\Phi_{\mathrm{P}}=0.3 .{ }^{1}$ All data was acquired following excitation at $425 \mathrm{~nm}$.

To account for inter-sample concentration variability, we made sure when possible (i.e. when an accetor was present in the sample) that the integrated emission intensity at direct excitation of Atto594 was similar for each sample used. Nevertheless, a high sample to sample emission variability was observed, thus we used another method to reduce the dependency on several samples. 

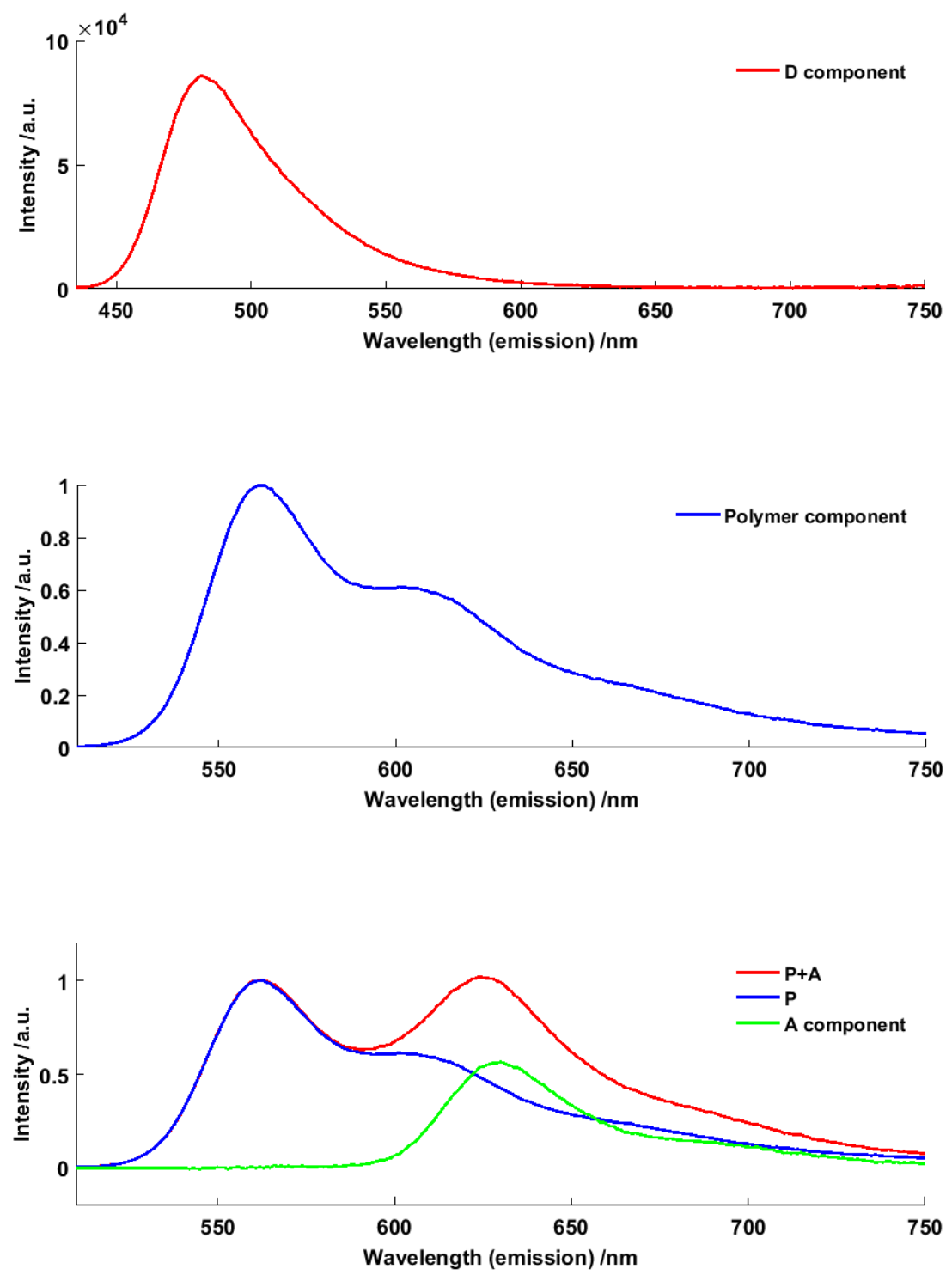

Figure S4. Examples of donor component spectrum (top), polymer component spectrum (middle), and acceptor component spectrum (bottom) obtained by subtracting polymer contribution from a polymer+acceptor spectrum.

In an alternative method, we determined all needed quantities from samples containing all three components (donor, polymer and acceptor). When exciting at $425 \mathrm{~nm}$, we spectrally decomposed acceptor emission, $I(A)$, from the measured emission spectrum. Similar to the previous method, we assumed that $I(A)_{D \rightarrow A}$ was negligible. $I(A)_{\text {direct }}$ was found by recording the acceptor emission intensity following direct acceptor excitation at $575 \mathrm{~nm}\left(I(A)_{A}\right)$ and correcting for differences in extinction coefficients for donor excitation at $425 \mathrm{~nm}$ and acceptor excitation at $575 \mathrm{~nm}$ using the acceptor absorption spectral information. 


$$
I(A)_{\text {direct }}=I(A)_{A} \cdot \frac{\epsilon_{425 \mathrm{~nm}}^{A}}{\epsilon_{575 \mathrm{~nm}}^{A}}
$$

$\varepsilon^{\mathrm{A}} 425 \mathrm{~nm} / \varepsilon^{\mathrm{A}}{ }_{575 \mathrm{~nm}}$ was determined from the absorption spectra of the acceptor as 0.12 . Likewise, $I(A)_{P \rightarrow A}$ was found by:

$$
I(A)_{P \rightarrow A}=\left(I(A)_{P}-I(A)_{A} \frac{\epsilon_{500 \mathrm{~nm}}^{A}}{\epsilon_{575 \mathrm{~nm}}^{A}}\right) \cdot \frac{\epsilon_{425 \mathrm{~m}}^{P}}{\epsilon_{500 \mathrm{~nm}}^{P}}
$$

Where $I(A)_{P}$ is the acceptor emission intensity following polymer excitation at $500 \mathrm{~nm}$. This quantity is a mix of emission due to excitation of the polymer and acceptor at $500 \mathrm{~nm}$. To account for the acceptor excitation, we subtract $I(A)_{A}$ (the acceptor emission following excitation at $575 \mathrm{~nm}$ ) corrected for differences in extinction coefficient of the acceptor at the two wavelength. $\varepsilon^{A} 500 \mathrm{~nm} / \varepsilon^{A}{ }_{575 \mathrm{~nm}}$ was determined from the absorption spectra of the acceptor as 0.09 . The obtained quantity is further corrected for the difference in extinction coefficients of poly(APPV-DNA) at $500 \mathrm{~nm}$ and $425 \mathrm{~nm} . \varepsilon_{425 \mathrm{~nm}}^{\mathrm{P}} / \varepsilon^{\mathrm{P}}{ }_{500 \mathrm{~nm}}$ was determined from the absorption spectra of poly(APPV-DNA) as 0.25 .

We determined the expected total donor emission by identifying all emission contributions from energy transfer after donor excitation and correcting for quantum yield differences between the different emitting species:

$$
I_{D t o t}=I(D)_{D}+\frac{\Phi_{D}}{\Phi_{P}} I(P)_{D \rightarrow P}+\frac{\Phi_{D}}{\Phi_{A}} I(A)_{D \rightarrow A}+\frac{\Phi_{D}}{\Phi_{A}} I(A)_{D \rightarrow P \rightarrow A}
$$

Where $I(D)_{D}$ is the donor excited donor emission intensity and $I(P)_{D \rightarrow P}$ is given by poly(APPV-DNA) emissions intensity following energy transfer from the donor. Direct excitation of the polymer $I(P)_{P}$ was taken into account using spectra taken following excitation at $500 \mathrm{~nm}$ where intensities were corrected for the difference in polymer extinction coefficient between the two excitation wavelength:

$$
I(P)_{D \rightarrow P}=I(P)_{D}-I(P)_{P} \frac{\epsilon_{425 n m}^{P}}{\epsilon_{500 n m}^{P}}
$$




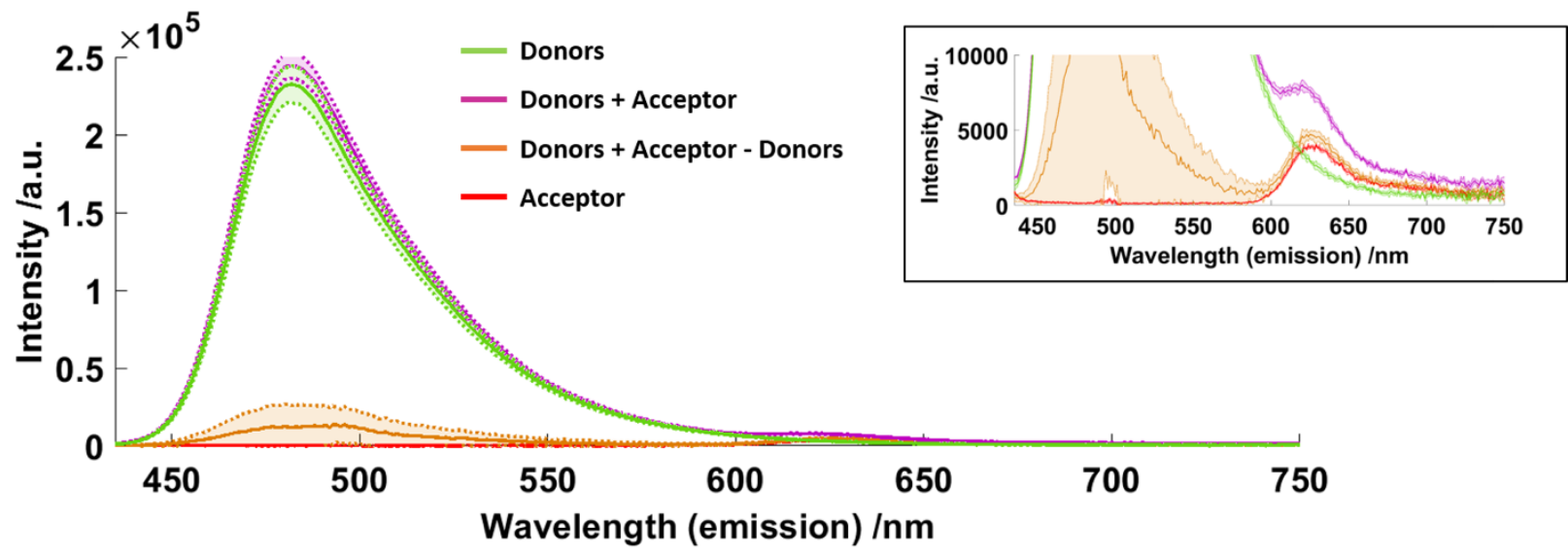

Figure S5. Spectra showing the emission spectrum for the indicated samples upon donor excitation. The orange spectrum is the result when subtracting the donor spectrum (green) from the spectrum for donors and the acceptor (purple). When comparing the acceptor signal from the acceptor alone (red) with the corrected spectrum (orange) it is clear that, the transfer from donors to the acceptor is miniscule. The scale is equal to Figure 2, while the inset shows a zoom. A mean of three triplicates is shown with the standard deviation. 

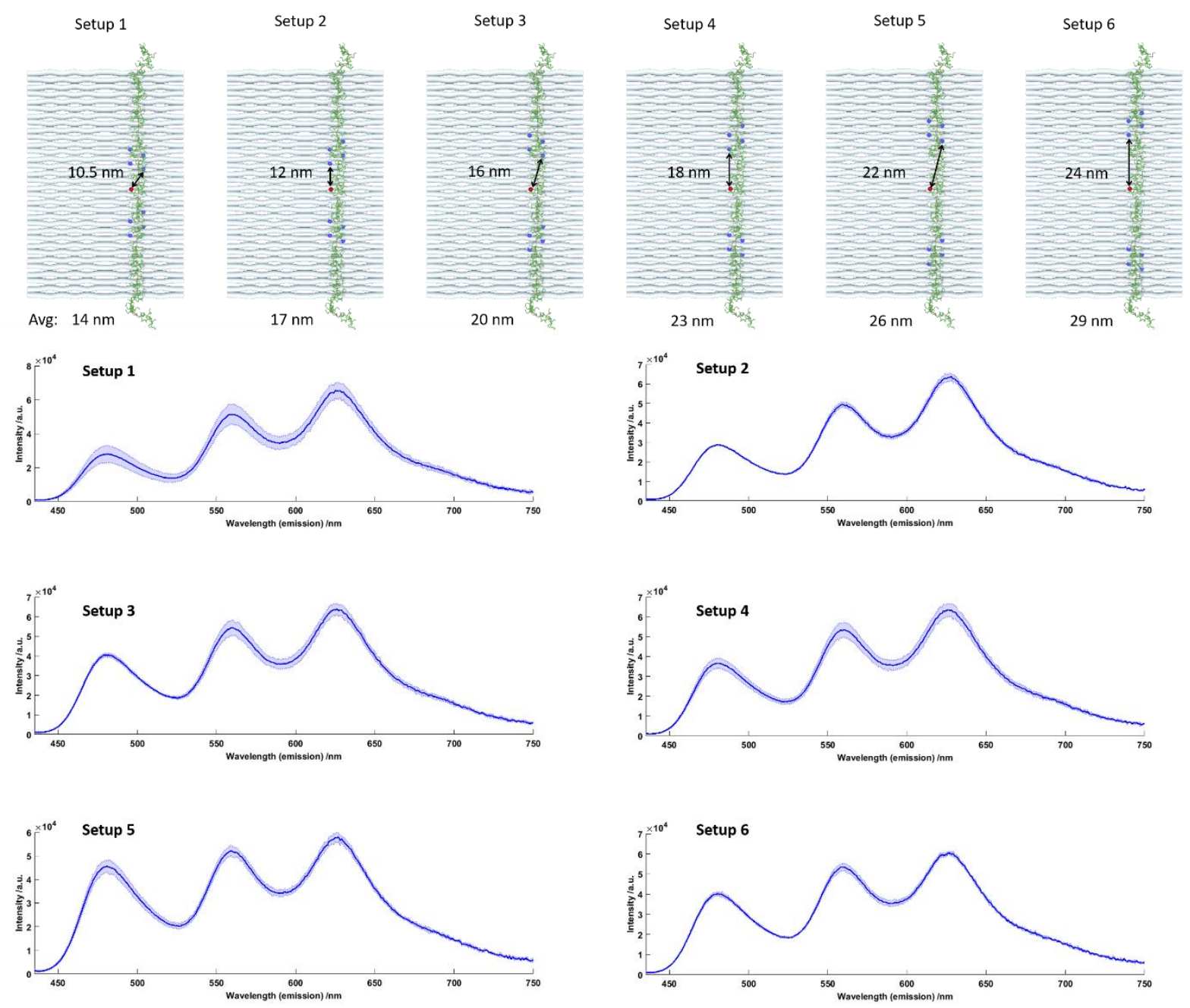

Figure S6. Top panel shows schematic illustrations of setup 1-6. The remaining part of the figure shows examples of average spectra from triplicates measured upon donor excitation. The bold blue lines show the mean spectrum from triplicates whereas the shade shows the standard deviation from the mean. 
$488 \mathrm{~nm}$

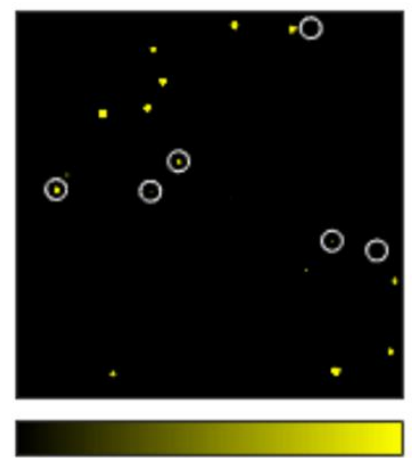

$488 \mathrm{~nm}$

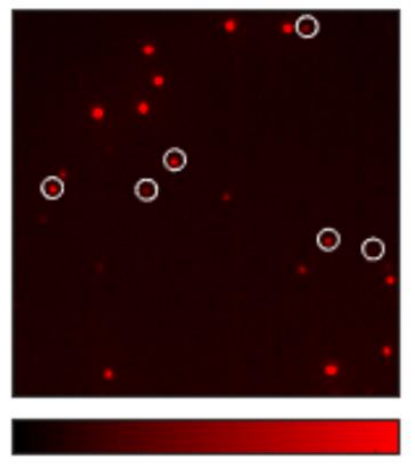

$150050 \quad 330 \quad 410480$

Figure S7. Example of fluorescence microscopy images of a DNA origami sample containing Atto425, poly(APPV-DNA) and Alexa647. The images show polymer emission (yellow image) and primarily acceptor emission (red images) for polymer excitation $(488 \mathrm{~nm})$. The white circles indicate fluorescence peaks from single origami constructs.

\section{S5. Single molecule microscopy data analysis}

Data analysis of single molecule fluorescence images (Figure S7) was performed with the iSMS software ${ }^{3}$ and custom scripts written in MATLAB. The peak finding algorithm in iSMS was used to identify fluorescent molecules. Single DNA origami constructs were identified when fluorescence from Alexa647 was observed to bleach or blink in a single step (Figure S8).

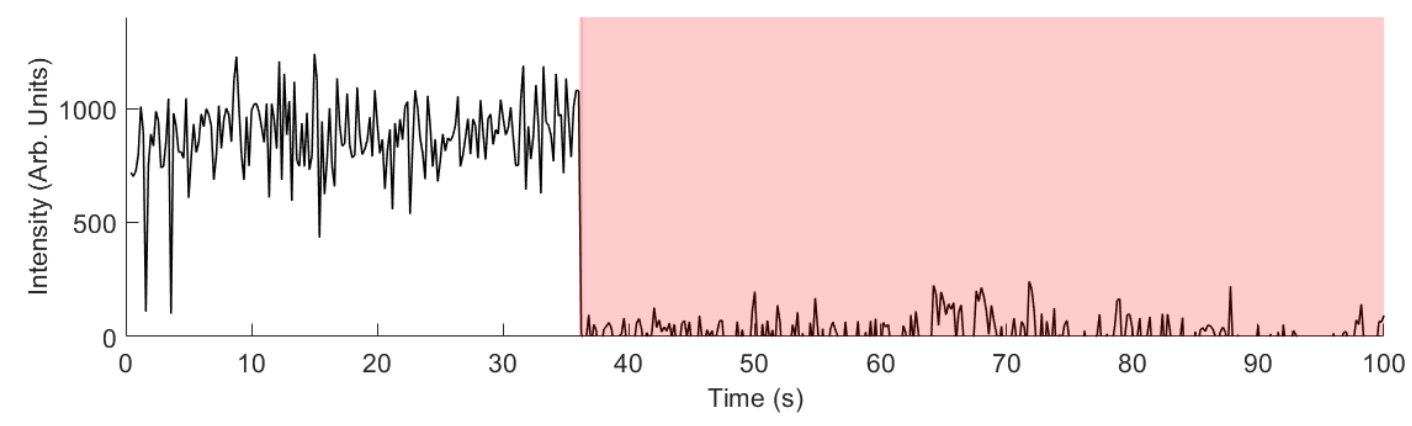

Figure S8. Example of a Alexa 647 fluorescence intensity time trace from a single DNA origami platform. The trace shows bleaching in a single step. This indicates that the fluorescence peak only contained one emitting Alexa647 fluorophore.

To further quantify energy transfer through the system on DNA origami samples containing all three components, we compared the fluorescence intensity in the red channel following excitation of Atto425 (at 405 $\mathrm{nm}$ ) and following direct excitation of poly(APPV-DNA) (at $488 \mathrm{~nm}$ ) (Figure 5a and S7). To achieve such comparable measures, we corrected the $488 \mathrm{~nm}$ excited fluorescence from the red channel for differences in excitation power and poly(APPV-DNA) absorption. This correction, $\varepsilon$, was determined directly from single 
molecule fluorescence as $\epsilon=\frac{I_{R(B)}^{405} \mathrm{~nm}}{I_{R(B)}^{488} \mathrm{~nm}}$ through the ratio of polymer fluorescence intensity in the red channel upon 405 and $488 \mathrm{~nm}$ excitation after bleaching of Alexa647 (Figure S9).
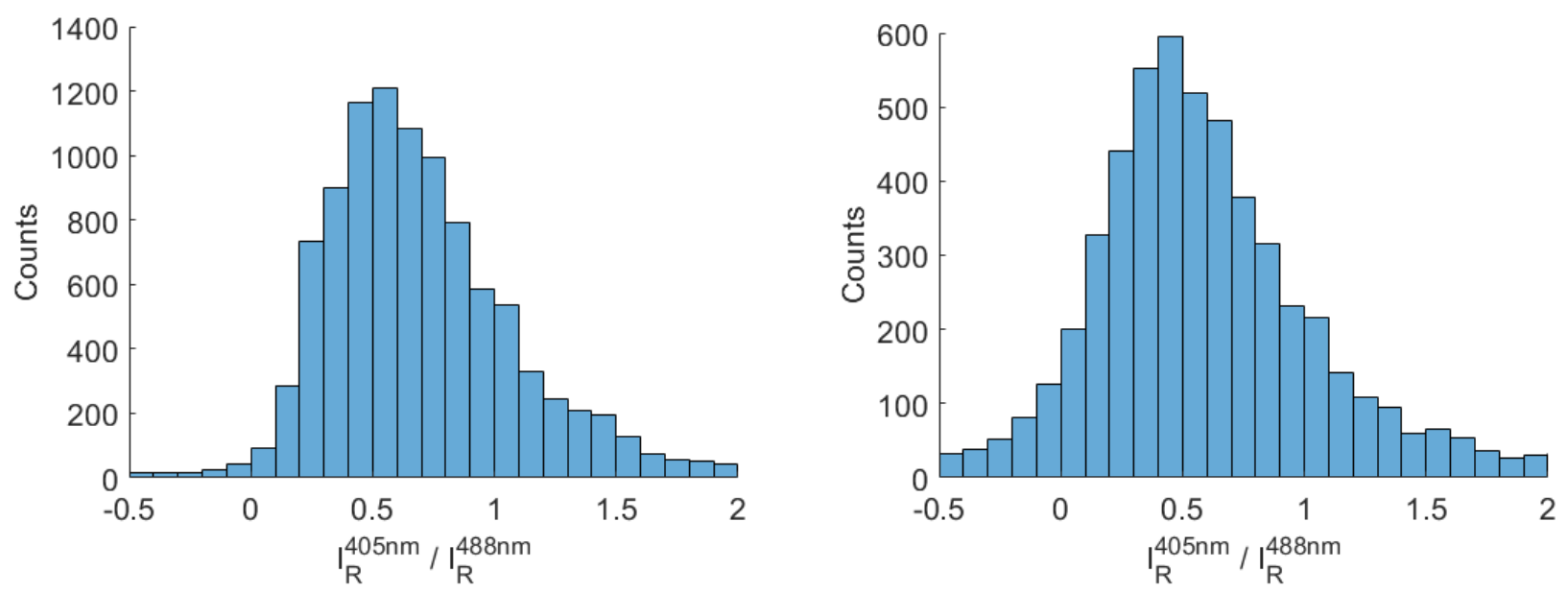

Figure 59. Histogram of $\epsilon$ determined from emission data of single molecules in a sample containing (left panel) Atto425, poly(APPV-DNA) and Alexa647, and (right panel) Poly(APPV-DNA) and Alexa647. The fluorescence intensity ratio was calculated for frames where Alexa647 had bleached. The $y$-axis shows the number of frames in absolute counts. Peak values are at $\epsilon=0.61$. (left panel) and $\epsilon=0.51$ (right panel). The histograms are based on 120 (left panel) and 75 (right panel) single molecules.

We find an overall agreement between $\varepsilon$ determined from single molecule data $(\varepsilon=0.61)$ and an estimate from ensemble absorption spectra while taking the experimental excitation powers at the different excitation wavelengths into account $\left(\varepsilon_{\text {ensemble }}=0.73\right)$. We note that after acceptor bleaching, Atto 425 might still absorb energy at $405 \mathrm{~nm}$ excitation and transfer it to poly(APPV-DNA). This might cause an overestimation of $\varepsilon$, since poly(APPV-DNA) would emit more in the red channel at $405 \mathrm{~nm}$ than $488 \mathrm{~nm}$ excitation. An overestimation of $\varepsilon$ would underestimate the effect of energy transfer through the whole system. We calculated the corrected red fluorescence intensity following excitation at $488 \mathrm{~nm}, I_{R c o r r}^{488}$, as:

$$
I_{R \operatorname{corr}}^{488 \mathrm{~nm}}=I_{R}^{488 \mathrm{~nm}} \cdot \epsilon
$$

Where $I_{R}^{488} \mathrm{~nm}$ is the fluorescence intensity in the red channel following excitation at $488 \mathrm{~nm}$.

If energy transfer proceeds through the system, we expect higher red fluorescence emission in the presence of donor excitation. We find that the corrected red fluorescence intensity is highest when the Atto425 donors are also excited (Figure S10, left panel). Both the integrated intensity and peak of the distribution are higher when exciting the system at $405 \mathrm{~nm}$. As a negative control, we did the same correction for experiments on samples containing only poly(APPV-DNA) and Alexa647 (figure S10, right panel). We find that in the absence of the donors, the corrected fluorescence intensities are comparable at the two excitation wavelengths. Taken together, this indicates energy transfer to the acceptor upon excitation of the donors. 

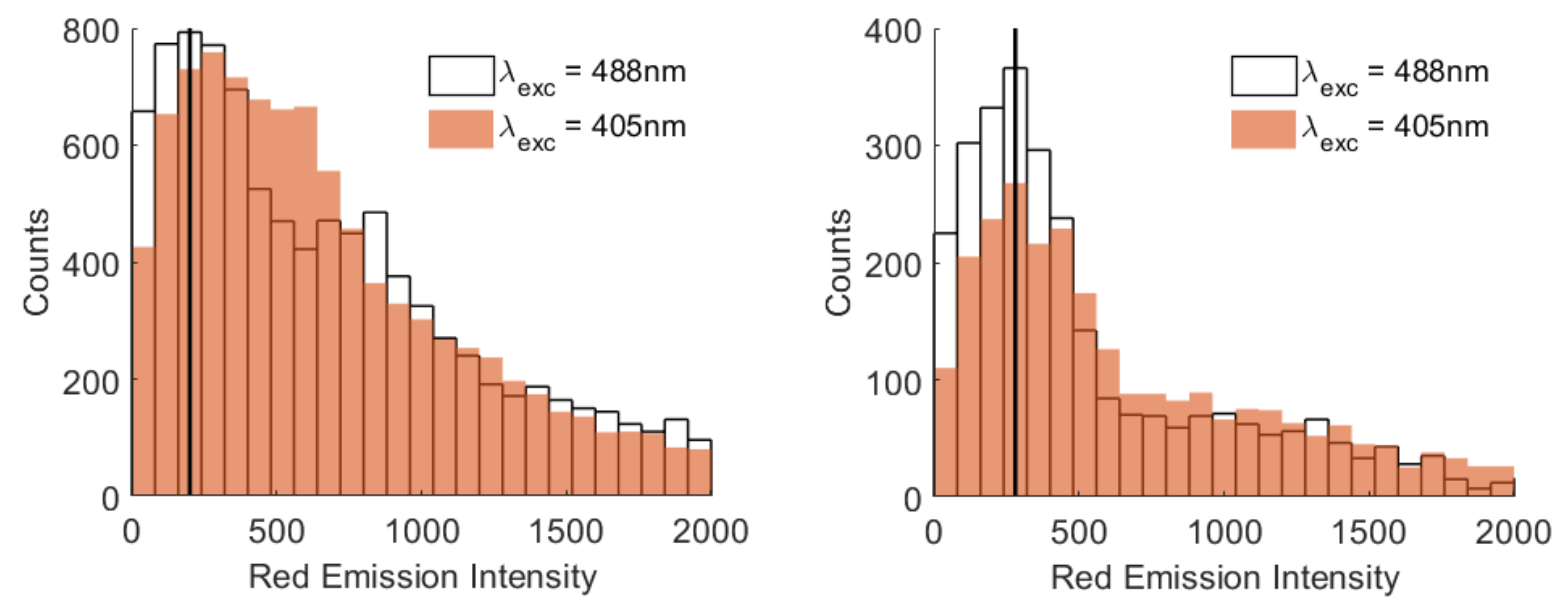

Figure S10: Red fluorescence intensity histograms of DNA origami samples containing (left panel) Atto425, poly(APPV-DNA) and Alexa647 and (right panel) poly(APPV-DNA) and Alexa647, excited at 488 (white) and $405 \mathrm{~nm}$ (orange), respectively. Fluorescence following excitation at $488 \mathrm{~nm}$ was corrected for differences in polymer excitation following equation 1 and $\varepsilon$ values from Figure $\mathrm{S} 8$. The vertical line marks the peak of the distribution for an excitation at $488 \mathrm{~nm}$. The histograms are based on fluorescence time traces from 120 (left panel) and 75 single (reight panel) origami constructs.

\section{S6. Estimation of polymer/origami ratio in ensemble studies}

In general, it is challenging to control the length of the polymer material during synthesis, and even after purification by size exclusion chromatography, the obtained poly(APPV-DNA) fractions show polydispersity. In our preliminary work, the length distributions of poly(APPV-DNA) was characterized by GPC as well as AFM. ${ }^{1}$ The AFM data showed lengths of polymers typically ranging from 20-200 nm in length with a small population of longer polymers. The length range of 20-200 nm corresponds to polymers containing 19-190 strands of ssDNA based on a repeating unit length of $0.665 \mathrm{~nm}$ and a degree of functionalization of 0.63 which was determined by $\mathrm{X}$-ray photon scattering (XPS) in our previous work. As a rule of thumb, we consider the average polymer chain in the experiments to have a length of around $100 \mathrm{~nm}$. With this length, the estimated number of polymer chains per origami rectangle should be 1.33. There are numerous uncertainties to this value, but it should provide a rough idea about the stoichiometry between polymer chains and DNA origami structures in the set-up.

\section{References}

\footnotetext{
${ }^{1}$ Knudsen, J. B.; Liu, L.; Bank Kodal, A. L.; Madsen, M.; Li, Q.; Song, J.; Woehrstein, J. B.; Wickham, S. F. J.; Strauss, M. T.; Schueder, F.; Vinther, J.; Krissanaprasit, A.; Gudnason, D.; Smith, A. A. A.; Ogaki, R.; Zelikin, A. N.; Besenbacher, F.; Birkedal, V.; Yin, P.; Shih, W. M.; et al., Routing of Individual Polymers in Designed Patterns. Nat. Nanotechnol. 2015, 10, 892-898.

${ }^{2}$ http://www.fluortools.com/software/ae (Accessed April 21, 2021)
} 
${ }^{3}$ Preus, S.; Noer, S. L.; Hildebrandt, L. L.; Gudnason, D.; Birkedal, V., iSMS: Single-Molecule FRET Microscopy Software. Nat Meth. 2015, 12, 593-594. 